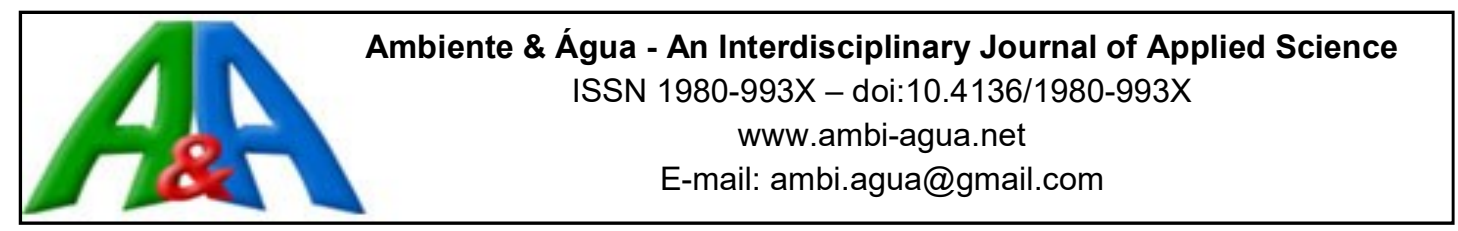

\title{
Toxicidade ambiental de efluentes advindo de diferentes laboratórios de uma farmácia magistral
}

\author{
doi:10.4136/ambi-agua.1761 \\ Received: 22 Sep. 2015; Accepted: 09 Jul. 2016 \\ Luciano Henrique Pinto*; Gilberto Cardozo; \\ Julia Carolina Soares; Gilmar Sidnei Erzinger \\ Universidade da Região de Joinville (UNIVILLE), Joinville, SC, Brasil \\ Laboratório de Fotoquímica e Fotobiologia \\ *Autor correspondente: e-mail: lucianohp.pq@gmail.com, \\ gilberto.cardozo@univille.edu.br, \\ julia-carolina16@hotmail.com,gerzinger47@gmail.com
}

\section{RESUMO}

Há uma crescente preocupação com os chamados "poluentes emergentes" na produção de medicamentos. Eles chegam ao meio ambiente a partir do descarte doméstico ou a partir das sobras geradas na produção de fármacos. Em pequenas concentrações da ordem de microgramas por litro ou inferiores, ou seja, concentrações não efetivas a curto prazo em termos de resposta biológica em seres humanos, podem causar grandes impactos no meio ambiente pela exposição crônica. O objetivo deste trabalho foi avaliar o risco de contaminação de efluentes pelo lançamento dos dejetos brutos da produção de medicamentos em pequena escala, por farmácias de manipulação, com base na avaliação da ecotoxicicidade pelo biomonitoramento de algas Euglena gracilis, e investigar os impactos apresentados sobre o comportamento e as alterações no processo de fotossíntese. Amostras de quatro laboratórios de diferentes demandas foram analisadas. As alterações comportamentais (velocidade de subida à superfície, $r$-value e velocidade de movimento) das algas foram biomonitoradas pelo NG-TOX e os parâmetros de fotossíntese foram medidos com o fluorímetro de pulso e amplitude modulada (PAM). Os resultados demonstraram que o efluente do laboratório de hormônios, que teve uma baixa produção semestral, apresentou pouco impacto, já o efluente do laboratório de psicotrópicos, mesmo com demanda intermediária, apresentou impacto significante sobre o comportamento e a atividade fotossintética das algas. A diferença de comportamento observada entre os diferentes setores de fármacos demonstra que os impactos e os possíveis riscos ambientais são diferentes para cada setor. A demanda e as diferentes substâncias manipuladas podem ser determinantes na classificação de risco e na escolha dos métodos de descontaminação.

Palavras-chave: biomonitoramento, descarte de medicamentos, ecotoxicidade, poluentes emergentes.

\section{Environmental toxicity of effluents of different laboratories of a compounding pharmacy}

\section{ABSTRACT}

There is a growing concern over so-called "emerging pollutants" in the production of pharmaceuticals. These pollutants reach the environment from household waste or from 
leftovers generated during production. In small concentrations of the order of micrograms per liter or less, or in concentrations ineffective at producing a biological response in humans in the short-term, chronic exposure can still have a great impact on the environment. This study evaluated the possible risk of contamination posed by the release of raw materials from smallscale drug production by compounding pharmacies, based on an ecotoxicity assessment conducted by biomonitoring Euglena gracilis algae. The study also investigated the impacts on behavior and the changes in the photosynthesis process of this algae. Samples of four laboratories with different demands were comparatively analyzed. Behavioral changes of the algae (ascent rate to the surface, r-value and speed of movement) were assessed by biomonitoring NG-TOX and photosynthetic parameters were measured by pulse-amplitude modulation fluorometer (PAM). The results showed that the effluent from hormone laboratory that had a low semestral production had little impact. On another hand, the effluent from the psychotropics laboratory, even with intermediate demand, had a significant impact on the behavior and photosynthetic activity of algae. The behavior differences observed between the different sectors of drugs shows that the impacts and potential environmental risks are different for each sector. The demand and the different substances manipulated can be crucial in risk classification and in the choice of decontamination methods.

Keywords: biomonitoring, disposal of medications, ecotoxicity, emerging pollutants.

\section{INTRODUÇÃO}

A questão da toxicidade ambiental vem causando preocupação na comunidade científica. Os estudos recentes nesta área permitiram a obtenção de resultados fundamentais para o direcionamento de outros trabalhos, bem como no desenvolvimento de processos de descontaminação de produtos agressivos ao meio ambiente. Isto implica em um cenário que obriga a revisão e reorientação de legislações vigentes que lidam com a questão ambiental, tanto em âmbito nacional quanto internacional (Kock-Schulmeyer et al., 2011).

Dentro da toxicidade ambiental, tem-se uma preocupação crescente em relação a "poluição emergente". O advento de tecnologias e outros processo facilitaram a identificação destes poluentes emergentes, e como o consumismo influenciou no acúmulo de substâncias no meio ambiente. Dentre tais produtos enquadrados na categoria de "poluição emergente", merece destaque a questão dos fármacos. Esses podem chegar ao meio ambiente a partir de descarte doméstico ou como resíduos de produção de fármacos produzidos em larga ou pequena escala, que acometem os recursos hídricos, o que não ocasionaria problemas de forma aguda, mas quando se trata de exposição crônica, alguns danos ambientais e de saúde para o homem podem ocorrer (Valcárcel et al., 2011b). Como exemplo de dano ao ambiente, podemos citar o estrógeno, hormônio feminino presente nos anticoncepcionais e nos medicamentos de reposição hormonal pós-menopausa, que pode afetar o sistema reprodutivo de organismos aquáticos, como os peixes machos que habitam ambientes contaminados, causando a feminização. Além disso, a contaminação por antibióticos pode contribuir para a resistência de bactérias, visto a capacidade de mutação presentes nesses organismos (Ueda et al., 2009).

Diferentes classes de antibióticos foram detectados simultaneamente no meio ambiente (Kolpin et al., 2004). As toxicidades individuais e combinadas de amoxicilina, eritromicina, levofloxacina, norfloxacina e tetraciclina foram examinados em dois organismos representativos do ambiente aquático: a cianobactéria Anabaena CPB4337, como um organismo alvo e a alga verde Pseudokirchneriella subcapitata. Mostrou-se que a combinação de eritromicina e tetraciclina apresentou um risco ecológico potencial para os organismos aquáticos com o padrão de uso atualmente de ambos os antibióticos (González-Pleiter et al., 
2013). Além disso, evidências sugerem que o betabloqueador propranolol tem efeitos no zooplâncton e nos organismos bentônicos (Valcárcel et al., 2011a). Outros efeitos também foram descritos em relação aos hormônios, como a feminização de peixes machos a partir da exposição ao estrógeno (Hugget et al., 2002).

No Brasil tem-se a Política de Recursos Hídricos que visa assegurar a adequada disponibilidade de água para consumo humano (Brasil, 1997) e a Portaria 2914/12, do Ministério da Saúde, que são documentos oficiais que definem os padrões de potabilidade para o consumo de água. Nestes documentos, por sua vez, não são abordadas as questões acerca dos fármacos (Cordeiro, 2009). Tal condição leva estes compostos a não serem identificados e nem sequer serem tratados nas estações de tratamento de água e de esgoto sanitário (D'acenzo et al., 2003). Sendo assim, no Brasil, o descarte de medicamento é regulado mas não de forma específica, e a abordagem com relação a toxicidade ambiental que pode acometer o meio ambiente não é considerada. Contrastando com a realidade brasileira, órgãos como a União Europeia (EU), a Agência de Proteção Ambiental do Norte da América (EPA) e a Organização Mundial da Saúde (OMS), já publicaram diretrizes e leis que visam alertar a respeito dos riscos da presença de medicamentos em águas e exigindo estudos que promovam a remoção destes, a fim de estabelecer limites aceitáveis para as águas disponíveis ao consumo humano (Esplugas et al., 2007).

Dentro de todo esse cenário, observa-se de maneira geral que processos convencionais de tratamento de água apresentam escassa utilidade na remoção deste tipo de poluentes, sendo esses encontrados em diversos rios. Nesse sentido, alguns trabalhos já são feitos com o intuito de identificar e remover medicamentos através de melhoramentos nos processos empregados. (Proia et al., 2013). Como exemplo, podemos citar estudos que avaliaram a remoção de estrógeno a partir da utilização de um sistema de filtro biológico em conjunto com o óxido de manganês ( $\mathrm{MnO} 2)$ e que obtiveram remoção de $81,7 \%$ de atividade, ou a partir de uma nova tecnologia, com recirculação e maior oxigenação (Balest et al., 2008; Rudder et al., 2004). Nos processos biológicos, por exemplo, a eficiência de degradação é fortemente influenciada pela presença de outros macroconstituintes, o que faz com que a degradação dos fármacos, além de ocasional, seja apenas parcial. Sistemas fundamentados em processos de adsorção têm sido recentemente propostos, utilizando-se sorbentes clássicos (carvão ativado) e modernos, como micelas pré-adsorvidas em montmorilonita (Cruz et al., 2010).

Além disso, na parte da identificação dos compostos destaca-se a cromatografia líquida acoplada à espectrometria de massa como técnica, que permite a identificação e a possibilidade de rastreamento dos micropoluentes orgânicos polares sem derivatização (Miao et al., 2002). A partir disso, em diversos países, são realizadas inúmeras análises afim de se estabelecer padrões de análise e verificar a qualidade das águas e efluentes. Essas análises demonstram que os resíduos de medicamentos em águas residuais e estações de tratamento de água são muito difundidos (Van der Ven et al., 2006; Zuccato et al., 2006). Entretanto, pouco se sabe sobre esses poluentes no Brasil (Raimundo, 2011).

Nesse contexto, as farmácias magistrais também geram resíduos potencialmente prejudiciais ao meio ambiente e considerados como "emergentes". Estudos realizados pela Agência Nacional de Vigilância Sanitária - ANVISA demonstraram que no Brasil são geradas cerca de 120 mil toneladas de lixo por dia, e que cerca de 1 a $3 \%$ desse total é produzido por estabelecimentos de saúde - incluindo as Farmácias Magistrais (estabelecimento de manipulação de fórmulas magistrais); sendo que cerca de $10 \%$ a $25 \%$ de resíduos em saúde representam risco ao meio ambiente e a saúde da população - incluindo os medicamentos (Brasil, 2012).

Diante desta realidade, estudos que visam identificar os resíduos e suas consequências no meio ambiente por meio de métodos eficazes são de suma importância, pois possibilitam extrapolar os resultados futuros destes contaminantes e promover ou estimular políticas 
eficientes de controle de descarte de resíduos na produção de medicamentos. Através de biomarcadores é possível identificar o risco de toxicidade ambiental de substâncias. Esses biomarcadores são microorganismos sensíveis a alterações ambientais e que podem emitir sinais de alerta a partir de mudanças comportamentais (Reviers, 2006).

Dentre esses microorganismos tem-se a Euglena gracilis, alga encontrada em ambientes dulcícolas, marinhos ou de águas salobras. São seres que não possuem parede celular, reproduzem-se assexuadamente e são protistas mixotróficos, ou seja, possuem hábito autotrófico, produzindo açúcares através da fotossíntese, ou heterotrófico, consumindo partículas alimentares por fagocitose. Esses organismos possuem dois flagelos com funções distintas, utilizando o flagelo longo para orientação e movimentação. Para observar o ambiente e procurar a melhor posição para a fotossíntese, a célula possui uma organela primitiva localizada na base do flagelo que filtra a luz solar em função de detecção de estruturas fotossensíveis. São seres que armazenam óleos e polissacarídeos como reserva, apresentando muitos plastos contendo clorofila a e b e carotenos. Essas algas apresentam características definidas quanto a sua mobilidade, velocidade de movimento, orientação espacial e flexibilidade. Assim, em um ambiente propenso ao seu desenvolvimento, estas algas possuem comportamento típico, porém em ambientes contaminados, um ou mais desses parâmetros citados podem sofrer alterações. Assim, a mudança de comportamento dessas algas torna-se um importante indicador de ecotoxicidade aquática (Reviers, 2006).

A fotossíntese das algas é outro parâmetro ecológico nos testes de bioensaios para monitorar a qualidade da água, uma vez que responde com sensibilidade às mudanças ambientais, através de uma variedade de mecanismos que influenciam a eficiência de captação de luz (Belshe et al., 2007). Desse modo, mudanças comportamentais e a eficiência fotossintética podem ser avaliadas através de equipamentos como o NGTOX (New Generation Ecotox) e o PAM. O primeiro apresenta a vantagem de ser um teste rápido, porém podem existir fatores como fluidez que possam afetar o alcance dos resultados, sendo então, nesse caso, requerido o uso do PAM como instrumento complementar de análise.

Portanto, este trabalho visa avaliar a toxicidade ambiental aguda que pode ser provocada por efluentes brutos, coletados de janeiro a julho de 2014, de diferentes laboratórios de uma Farmácia Magistral via NGTOX e PAM, para assim colocar em pauta questões de possíveis riscos e subsidiar discussões sobre a necessidade de revisão da legislação vigente que aborde as questões ambientais. Objetiva também validar o uso do NGTOX isoladamente para análises rápidas e em tempo real.

\section{MATERIAIS E MÉTODOS}

Este estudo foi realizado no Laboratório de Fotoquímica e Fotobiologia da Universidade da Região de Joinville.

Trata-se de um estudo experimental, envolvendo o uso de algas do gênero E. gracilis KLEBS obtidas da coleção da Universidade de Gottingan, Alemanha. Em relação às algas, foram avaliadas às alterações comportamentais e a atividade fotossintética quando submetidas ao contato com água de acesso a farmácia, bem como com os efluentes brutos.

Para a realização do estudo contou-se com a colaboração de uma Farmácia de Manipulação da cidade de Joinville, norte de Santa Catarina, que permitiu a coleta das amostras dos quatro ambientes de produção da farmácia: O "laboratório de psicotrópicos", responsável pela manipulação de formulações dos medicamentos classificados pela portaria 344/98 de venda controlada; o "laboratório de hormônios", responsável pelas manipulações de formulações restritamente hormonais; o "laboratório de sólidos", responsável pela produção dos encapsulados e das demais formulações sólidas e o "laboratório de dermato", responsável pela produção das formulações sólidas e semi-sólidas de propriedades 
dermatológicas.

As amostras tomadas como "água de acesso" foram coletadas diretamente das torneiras das pias destinadas à lavagem do material de cada laboratório, utilizando frascos de vidro borossilicato previamente esterilizados - as quais foram utilizadas como "controle", e as amostras tomadas como "efluentes brutos" foram coletadas dos sifões ligados às pias de lavagem dos materiais por meio de uma bomba peristáltica. Nesta coleta também foram utilizados frascos de borossilicato esterilizados. Todas as coletas retiraram alíquotas de 125 $\mathrm{mL}$. Após as coletas, as amostras foram guardadas em caixas de isopor com gelo e ao abrigo da luz.

Os testes foram realizados com algas Euglena gracilis em meio mineral e orgânico preparado conforme descrição feita por Checcuci et al. (1976). A manutenção da cultura ocorreu sob exposição da luz $20 \mathrm{~W} / \mathrm{m}^{2}$, foto período de $12 \mathrm{~h}$ a $20^{\circ} \mathrm{C}$. Todos os testes foram feitos a partir de uma única cultura, que foi fracionada com o intuito de manter as características e evitar desvios relativos a preparos diferentes de meio mineral. O estudo se baseia no trabalho desenvolvido por Häder e Lebert (1985), aperfeiçoado por Erzinger et al. (2011) sendo reproduzido em diversos trabalhos pelo mundo como os realizados por Ekelund e Nilsson (2008).

As culturas preparadas nas condições descritas anteriormente foram mantidas em uma única cultura de $300 \mathrm{~mL}$, tratadas durante um período de uma semana a fim de se manter homogeneidade do meio para a aplicação dos testes futuros. Esta cultura foi subdivida em 6 frações de $50 \mathrm{~mL}$, as quais receberam posteriormente a adição da solução das amostras de água de abastecimento e efluentes (amostras testes). A adição das amostras atendeu ao seguinte procedimento: inicialmente foi retirado $0,5 \mathrm{~mL}$ de cada cultura, para a adição em seguida de $0,5 \mathrm{~mL}$ da solução contendo as amostras testes, perfazendo um volume final de $50 \mathrm{~mL}$ para cada cultura. As algas ficaram em contato com as amostras de efluentes por 1 mês, conforme recomendação de Ekelund e Nilsson (2008) para depois serem analisadas no NGTOX e PAM (Figura 1). Em todos os testes, o pH das amostras era de 6,0 a 7,0.

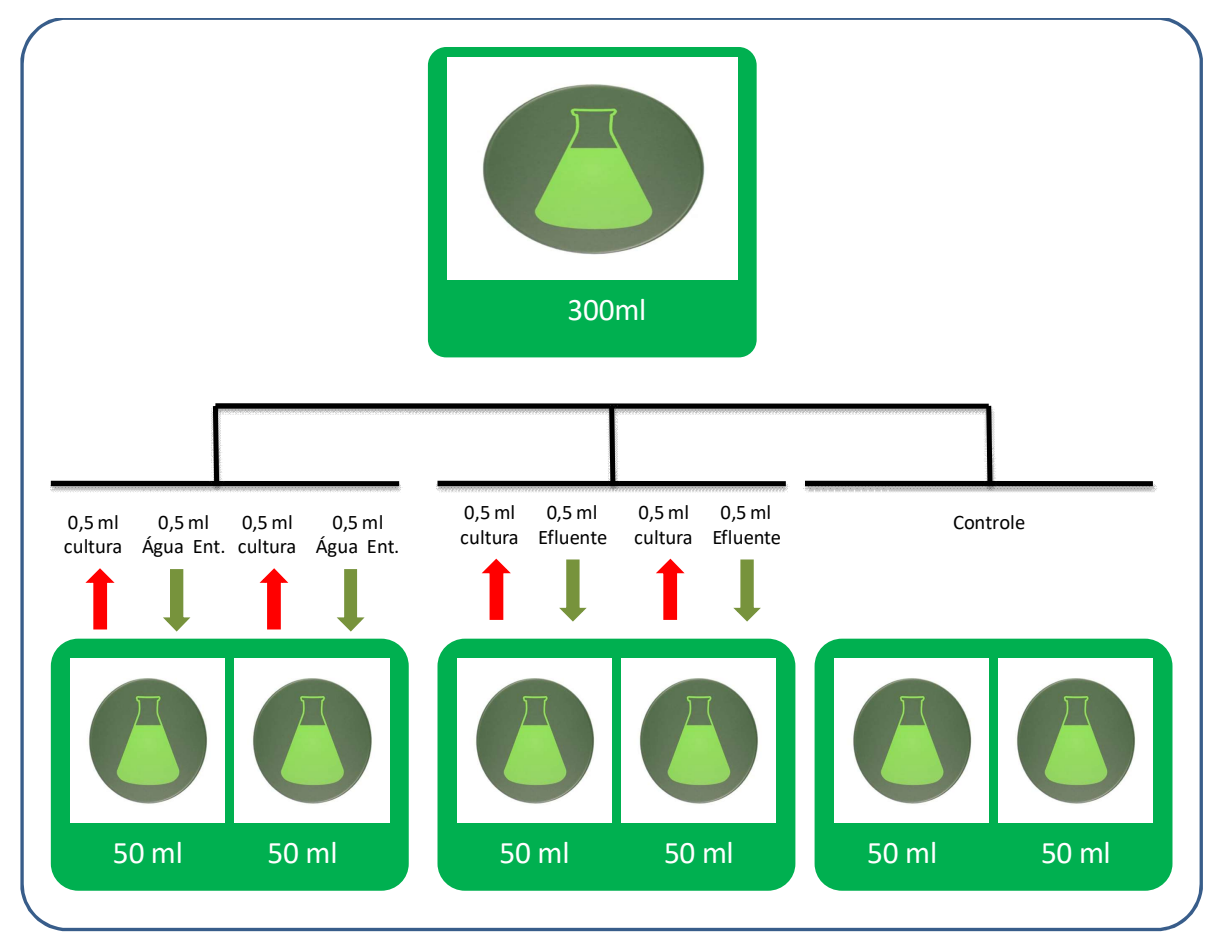

Figura 1. Esquema de preparação das culturas de algas. 
Os experimentos comportamentais com Euglena gracilis na presença de amostras da água de acesso e dos efluentes - utilizando-se $20 \mathrm{~mL}$ desses efluentes em diluição 1:1 - foram então realizados via NG-TOX (Figura 2), equipamento desenvolvido e homologado pela Ecobabitonga Tecnologia Ltda (Erzinger et al., 2011). A ferramenta monitorou, através de análise de imagem em tempo real, o comportamento das algas, usando diferentes parâmetros de movimento do flagelado unicelular fotossintetizante.

O equipamento é constituído por um sistema de conexões envolvendo quatro tubos de silicone responsáveis pela sucção da (1) cultura de células de E. gracilis, (2) amostra de águas contendo efluentes para o teste, (3) água para diluição das amostras e (4) descarte do material analisado. Três bombas acionadas por motores de passo peristáltico transportam as células, $o$ diluente e a amostra até uma cubeta de vidro de $22 \mathrm{~mm}$ de diâmetro interno e 0,2 $\mathrm{mm}$ de espessura. Os organismos teste, em contato com o diluente, são homogeneizados e enviados para uma cubeta de observação conectada a um microscópio, que captura as imagens das células em movimento (Figura 2). As imagens são gravadas por uma câmara CCD (charged coupled device) e digitalizadas por uma placa conectada a um microcomputador, onde são apresentadas em um monitor. O software então calcula os parâmetros de movimento, velocidade de locomoção, velocidade de subida a superfície, tamanho médio das células; entre outros parâmetros (Häder e Lebert, 1985). Em seguida é feita a adição das amostras e após um período de 10 minutos é refeita a análise dos parâmetros pelo software. Qualquer alteração nos movimentos, velocidades médias, velocidades de súbida a superfície e tamanho celular são calculadas e comparadas com o resultado anterior.

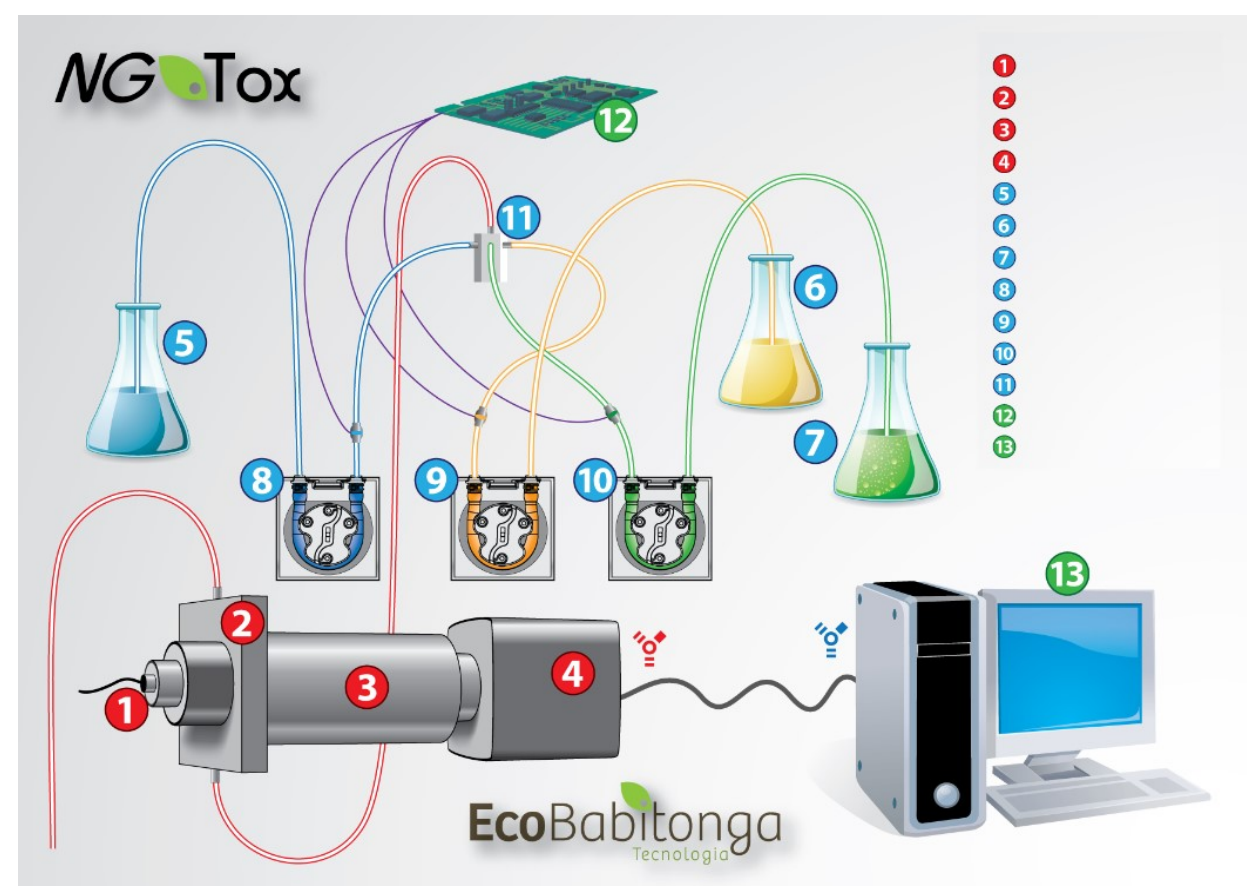

Figura 2. Esquema de funcionamento do NG TOX. Autorizada por Erzinger et al. (2011).

Os parâmetros de fotossíntese foram medidos através de um fluorímetro de amplitude de pulso modulada PAM 2000, walz, Effeltrich, Alemanha. O princípio de medição do PAM é baseado em mudanças no nível de fluorescência da clorofila, após a aplicação de pulsos de luz saturada. $\mathrm{O}$ rendimento de fotossíntese bem como de comportamento (fotoquímico e não fotoquímico) foram então calculados. 
As culturas testadas foram adaptadas na ausência de luz durante $1 \mathrm{~h}$, sendo que em seguida foram retirados cerca de $5 \mathrm{~mL}$ e transferidos para cubeta do equipamento PAM. Foi então submetida à emissão dos pulsos de luz saturante para avaliação da atividade fotossintética.

A emissão de um pulso de luz saturante permitiu detectar a fluorescência máxima Fm, indicando redução completa do receptor de elétrons FSII. A Luz-resposta das curvas foi determinada para todas as amostras tratadas. As algas foram expostas a intensidade da iluminação crescente (gerado por uma lâmpada halógena interna) em 10 passos de 0 a $3111 \mathrm{~mol} \mathrm{~m}^{-2} \mathrm{~s}^{-1}$ ). Após $20 \mathrm{~s}$ de cada etapa de iluminação, um pulso saturante foi aplicado e o rendimento fotossintético e a ETR (taxa de transporte de elétrons) foram medidos automaticamente.

Em seguida, os dados foram plotados contra o PAR incidente (radiação fotossinteticamente ativa em $\mathrm{mol} \mathrm{m} \mathrm{m}^{-2} \mathrm{~s}^{-1}$ ). Desta forma, foi feita a interferência que as amostras promoveram na Eficiência Global Fotossintética. As análises estatísticas do comportamento das algas avaliadas no NG-TOX e PAM foram realizadas utilizando-se o aplicativo ImagingTox ${ }^{\circledR}$. O ImagingTox ${ }^{\circledR}$ permite a realização de análise estatística 5-PL integrada.

\section{RESULTADOS E DISCUSSÃO}

Nota-se uma produção mais acentuada de formulações denominadas "sólidas", nas quais se incluem cápsulas utilizadas oralmente. Elas representam mais da metade de todas as formulações produzidas no período avaliado. Em segundo lugar as formulações de "dermato", nas quais se tem cremes em que são incorporados diversos ativos. Em menor quantidade temos as formulações da portaria 344/98, que segundo a legislação vigente exige um laboratório a parte, assim como o dos hormônios, que pelo risco de contaminação também exige aparatos especiais para a manipulação destas substâncias (Tabela 1).

Tabela 1. Número de formulações produzidas de janeiro a julho de 2014.

\begin{tabular}{|c|c|c|}
\hline Laboratório & $\begin{array}{l}\mathrm{N}^{\circ} \text { de formulações dos } \\
\text { últimos } 06 \text { meses }\end{array}$ & $\begin{array}{c}\text { Representação } \\
\text { (em porcentagem) }\end{array}$ \\
\hline Psicotrópicos & 3150 & 18,6 \\
\hline Hormônios & 728 & 4,3 \\
\hline Sólidos & 9388 & 55,5 \\
\hline Dermato & 3638 & 21,5 \\
\hline Total & 16904 & 99,9 \\
\hline
\end{tabular}

Fonte: Registros internos da farmácia.

Em testes realizados via NGTOX, todas as amostras contendo algas submetidas ao contato com os efluentes brutos analisadas apresentaram algum tipo de alteração comportamental quando comparada aos controles - amostras que continham apenas algas sem contato com efluentes. Dentre essas alterações comportamentais apresentadas, a única que apresentou resultados significativos foi o de inibição do comportamento r-value, condição essa que pode ser impactante para a atividade fotossintética e apresentar risco ambiental. As águas de acesso, provenientes do serviço de tratamento e coletadas dentro da própria farmácia, não levaram as algas a apresentarem alterações comportamentais relevantes quando comparadas ao controle que continha apenas algas em meio de cultura padrão. 
Observou-se no teste de alterações comportamentais das algas que o laboratório de sólidos, que representa 55,5\% da produção semestral, apresentou uma inibição de r-value de $20 \%$, um comportamento semelhante ao apresentado pelo laboratório de psicotrópicos, que apresentou inibição de aproximadamente $24 \%$ neste comportamento das algas. Porém vale ressaltar que este último laboratório, em comparação com o de sólidos, apresenta produção menor $-18,6 \%$ da produção total no mesmo período (Figura 3).

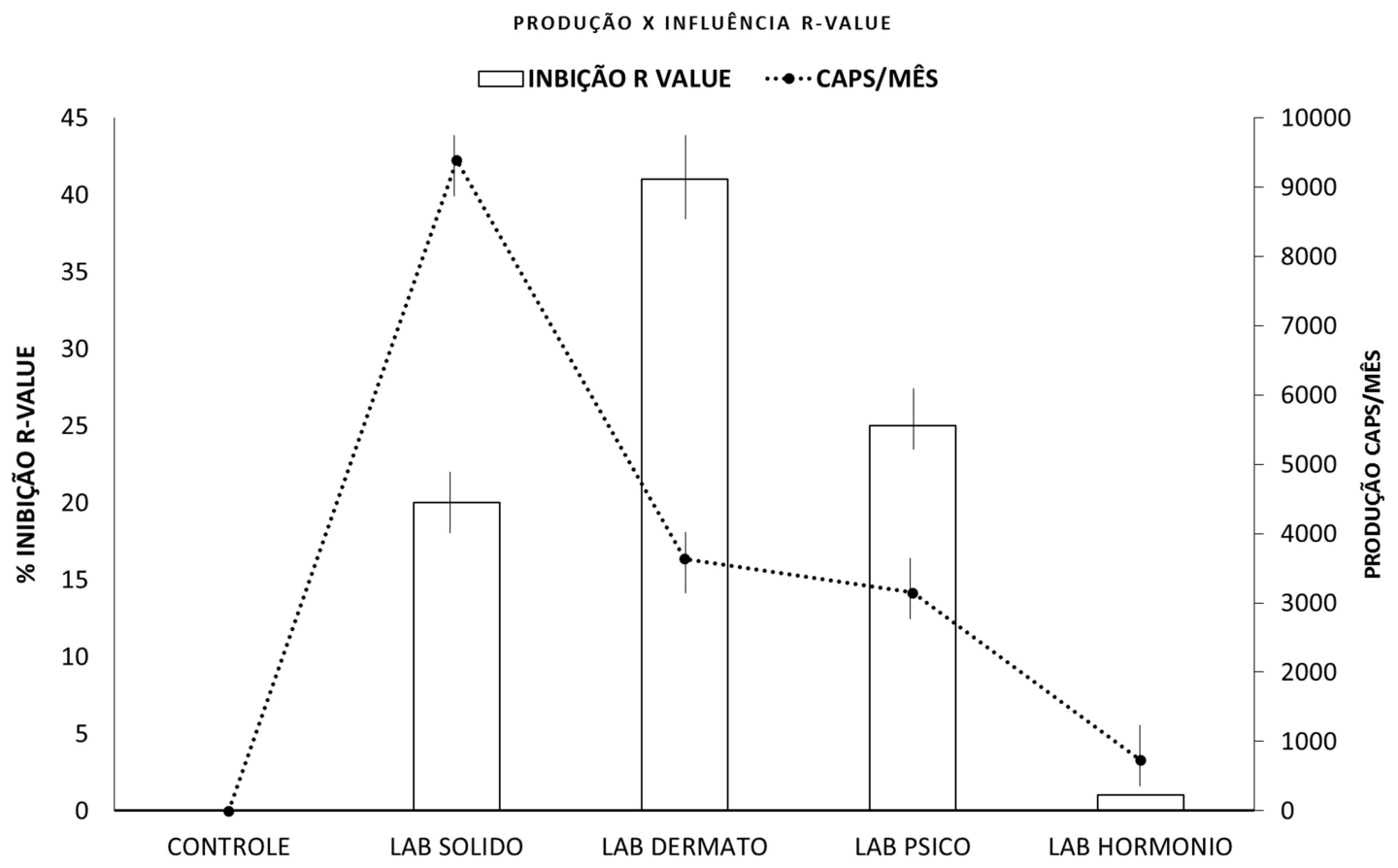

Figura 3. Relação entre a porcentagem de inibição x produção por laboratório.

O maior índice de inibição de r-value observado ficou por conta das análises realizadas nas amostras dos efluentes do laboratório de dermato; este demonstrou uma inibição de aproximadamente $42 \%$ e teve uma produção semestral de 3638 formulações, o que representa apenas $21,5 \%$ da produção total dos últimos seis meses, sendo o que mais produziu e consequentemente contribuiu para uma maior concentração de contaminantes nos efluentes. $\mathrm{O}$ laboratório de hormônios, que teve uma baixa representação na demanda semestral, também apresentou uma baixa inibição de r-value - cerca de $2 \%$ de inibição quando comparado ao controle.

As amostras do laboratório de sólidos, responsável pela segunda menor inibição de rvalue $(20 \%)$ demonstraram também, no teste de Eficiência Fotossintética no PAM, a segunda menor inibição da atividade fotossintética, apresentando um índice de EGF de 0,4 Fm/min. Apesar dessas alterações, esse laboratório demonstrou, em relação aos laboratórios de dermato e hormônios, causar menor impacto ao microrganismo analisado nos ensaios de exposição aguda. A grande maioria dos estudos envolvendo o descarte de medicamentos apontam os AINEs, hormônios e psicoativos como as principais classes encontradas no ecossistema analisado. Segundo estudo conduzido por Santos et al. (2010), os AINES, seguido pelos antibióticos, agentes dislipidêmicos e hormônios são as principais classes envolvidas em episódios de contaminação do ambiente por químicos de utilização terapêutica. Porém, se extrapolarmos a quantidade do laboratório de hormônio, que obteve o menor índice nos testes executados, para a mesma quantidade de formulações do laboratório de sólidos teríamos, para as análises de hormônios, $25,79 \%$ de inibição de r-value reforçando a hipótese 
do laboratório de sólido possuir a menor taxa de impacto ambiental dentre as demais classes analisadas.

O ensaio de eficiência global fotossintética (EGF) apresentou resultados diferentes para as amostras dos laboratórios estudados (Figura 4), o que demonstra que não foi apenas uma questão de alteração de viscosidade, mas sim de eficiência fotossintética. A fotossíntese foi comprometida mais significativamente pelas amostras dos laboratórios de sólidos, dermato e psicotrópicos.

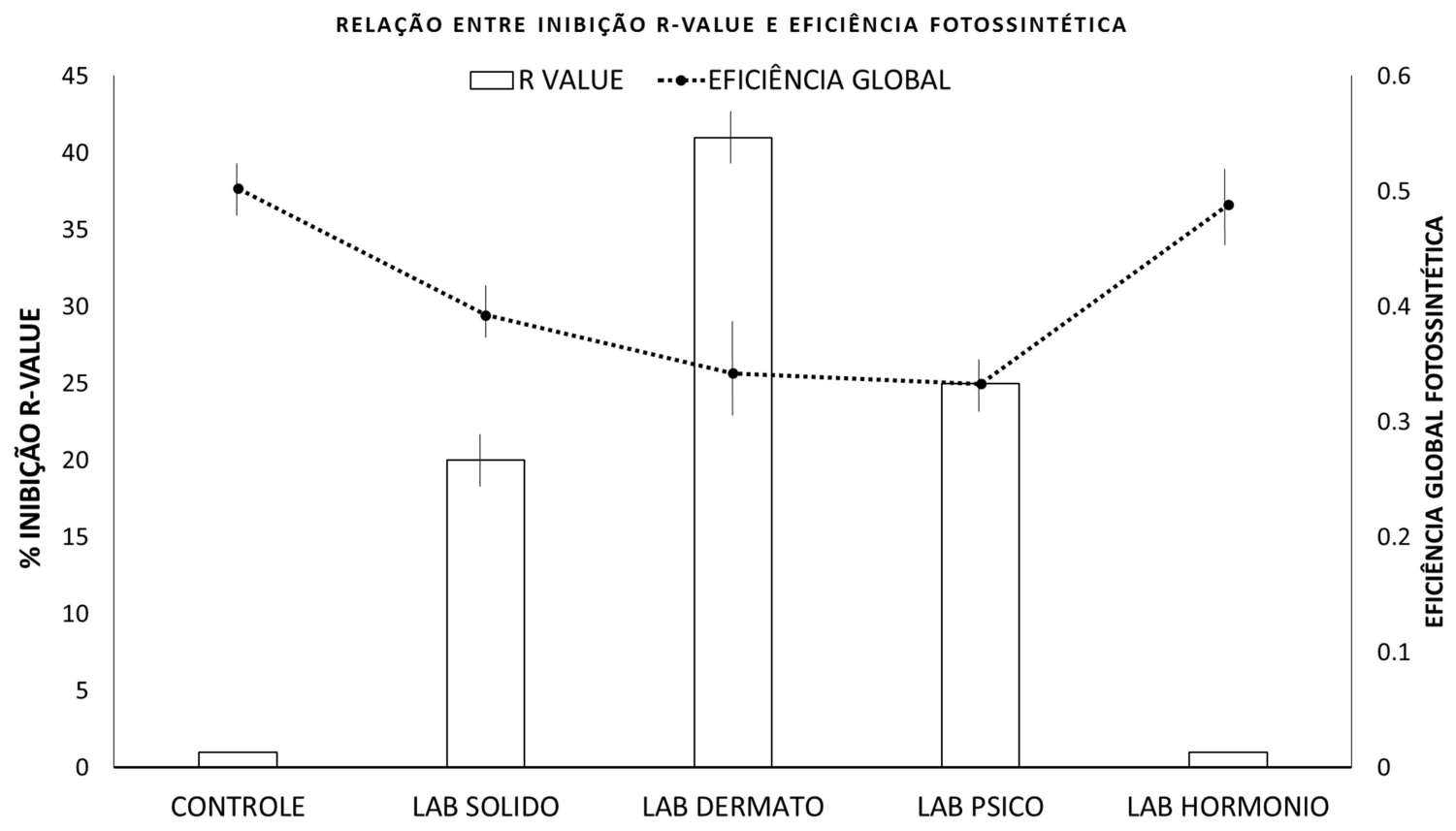

Figura 4. Porcentagem de inibição de r-value $X$ eficiência global fotossintética.

O laboratório de psicotrópicos que teve uma inibição superior com r-value de $25 \%$ no teste anterior, nesta etapa se comportou de forma diferente e apresentou a maior inibição fotossintética dentre as amostras analisadas, com EGF de 0,33 Fm/min (Tabela 2), evidenciando que a quantidade aqui não foi o determinante para a redução da eficiência, mas sim a natureza dos compostos encontrados nos efluentes. Esses resultados refletem a condição que existem substâncias que influenciam o r-value por exercerem alguma atividade biológica dentro das algas, modulando canais de membrana que resultariam na redução do alcance do potencial de ação o que provavelmente dificultaria o alcance da luz e consequentemente a inibição na EGF acaba sendo mais impactante. Além disso, segundo o Manual de Farmacologia e Terapêutica de Brunton et al. (2012), a grande maioria dos psicotrópicos é constituída por moléculas altamente lipofílicas, o que permite uma rápida penetração nas membranas celulares, resultando em uma rápida absorção e distribuição e maior acesso ao sistema nervoso central. Acredita-se que o aumento na oferta de aporte energético com a incorporação dos lipídios presentes nas amostras desse laboratório possa ter influenciado no processo de fotossíntese de maneira similar ao ocorrido com as amostras do laboratório de dermato.

De forma semelhante ao ocorrido no ensaio via NGTOX no qual apresentou a maior alteração comportamental com inibição de r-value de $42 \%$, as amostras obtidas do laboratório de dermato, apresentaram um alto índice de comprometimento da eficiência global fotossintética, ficando com a segunda maior inibição do teste no PAM, com EGF de $0,35 \mathrm{Fm} / \mathrm{min}$ (Tabela 2). Esses resultados refletem à questão do aumento da oferta de lipídeos 
contidos nas amostras desse laboratório, que podem ter influenciado na fotossíntese.

Tabela 2. Eficiência global fotossintética por laboratório.

\begin{tabular}{lc}
\hline Amostras & $\begin{array}{l}\text { Eficiência Global } \\
\text { Fotossintética (EGF) }\end{array}$ \\
\hline Controle & $0,51 \mathrm{Fm} / \mathrm{min}$ \\
Lab. Hormônios & $0,49 \mathrm{Fm} / \mathrm{min}$ \\
Lab. Sólidos & $0,41 \mathrm{Fm} / \mathrm{min}$ \\
Lab. Dermato & $0,35 \mathrm{Fm} / \mathrm{min}$ \\
Lab. Psicotrópicos & $0,33 \mathrm{Fm} / \mathrm{min}$ \\
\hline
\end{tabular}

O laboratório de hormônios, que teve influência quase nula na análise comportamental também não apresentou uma influência importante na atividade fotossintética das algas com uma inibição muito próxima da inibição promovida pelo controle - uma EGF de $0,48 \mathrm{Fm} / \mathrm{min}$ contra $0,51 \mathrm{Fm} / \mathrm{min}$ do controle. Isso pode ser explicado pelo fato das algas $E$. gracilis serem seres que armazenam óleos e polissacarídeos como reserva energética, apresentando muitos plastos contendo clorofila A e B e caroteno (Reviers, 2006), e com o aumento na fonte de carbono estas substâncias lipofílicas poderiam se acumular no interior destas algas e influenciar no comportamento destas, seja por biomagnificação, seja por ocupar lugar destinado a substâncias importantes em seu metabolismo, ou ainda, com um maior aporte energético a busca pela obtenção de energia pelo processo de fotossíntese pode ter sido inibida. Estudos conduzidos por Ekelund e Nilsson (2008) utilizando E. gracilis na presença de compostos estrogênicos apontaram que estas algas foram sensíveis à exposição de compostos lipofílicos.

Além disso, outra hipótese que justifica a baixa inibição apresentada nas amostras do laboratório de hormônios pode ser atribuída à demanda de produção semestral pequena (cerca de 4,3\% da produção total), o que inferiu à amostra estudada uma baixa concentração dos hormônios. Estes resultados contrapõem aos estudos feitos por Pinto (2012) no qual outros parâmetros como "velocidade de subida a superfície" e "mobilidade" foram afetados, e apresentaram ser efeitos dose dependentes. Isto aponta para a possibilidade de que a demanda então produzida não apresenta riscos ambientais nítidos. No teste de EGF teve-se a confirmação da pouca influência dos resíduos de hormônios - na concentração encontrada nas amostras - sobre a fotossíntese. Os valores $0,49 \mathrm{Fm} / \mathrm{min}$ da amostra contra $0,51 \mathrm{Fm} / \mathrm{min}$ do controle suportam essa afirmação. O estudo de biomonitoramento desenvolvido por Pinto (2012), e já citado anteriormente, demonstrou a existência de uma influência dos hormônios $17-\alpha$-estradiol e 17- $\beta$-estradiol sobre o comportamento das algas E. gracilis.

Desse modo, observou-se alterações no comportamento das algas (mobilidade, velocidade de movimento, orientação espacial e flexibilidade), obtido pelo biomonitoramento via NGTOX, sendo o r-value o mais significativo e presente em todas as amostras testadas. A alteração de r-value implica em perda da uniformidade dos movimentos do conjunto de algas, o que pode influenciar outros parâmetros de interesse ecotoxicológico, como a eficiência fotossintética, que se alterado, aponta um risco ambiental, já que indica que há uma mudança no comportamento desses animais (Belshe et al., 2007). As análises foram direcionadas simulando uma exposição aguda. Buscou-se neste primeiro momento verificar se haveriam impactos iniciais que fossem significativos e sugestivos de novas análises e testes de exposição crônica. A importância de tal conduta reside no fato da necessidade de testes rápidos, que possam remediar efeitos deletérios o quanto antes (Cordeiro, 2009). O teste de 
eficiência global fotossintética buscou, em um segundo momento, identificar a influência das amostras analisadas sobre a atividade fotossintética das algas E. gracilis.

Outro fator observado demonstra que não existe uma relação direta entre as alterações encontradas sobre os valores de r-value e o decréscimo da EGF. Em algumas situações a alteração de r-value pode ter sido influenciada fisicamente pela viscosidade da amostra, comprometendo a dispersão das algas no meio, porém tendo discreta influência no processo de fotossíntese, uma vez que, as amostras do laboratório de dermato que apresentou maior valor de inibição do r-value teve menor inibição na EGF.

Uma outra condição que poderá ser observada e melhorada futuramente, a fím de promover maior representatividade da amostra, diz respeito ao método de coleta que poderá ser realizada em diferentes períodos, assim os dados de cada período poderão ser analisados e comparados e, com tratamento estatístico, apresentar números com maior fidelidade.

\section{CONCLUSÃO}

Os resultados obtidos com o presente estudo possibilitam diversas análises e prospecções a partir do estudo do risco de ecotoxicidade e do biomonitoramento de efluentes advindos de farmácia de manipulação. Observou-se nas quatro amostras analisadas - cada uma proveniente de laboratórios diferentes (hormônios, sólidos, dermato e psicotrópicos), contendo efluentes brutos dos laboratórios, uma interferência no comportamento r-value das algas $E$. gracilis quando comparadas ao controle de água tratada antes de entrar no processo.

A diferença de comportamento observada entre os setores de produção da farmácia demonstra que os impactos e os possíveis riscos ambientais são diferentes para cada setor. A demanda de cada setor e as diferentes substâncias manipuladas podem ser determinantes na classificação de risco e na consequente necessidade e escolha dos métodos de descontaminação. Os fatores de impacto por classe e demanda são preponderantes para avaliarmos o risco ambiental. A utilização de métodos que identificassem qual seria a concentração limite de cada setor seria de grande importância para orientar o tratamento destes efluentes. Uma vez determinados estes valores de ponto de risco de cada setor, a utilização do NGTOX como ferramenta de identificação pela praticidade, fidelidade e rapidez dos testes, seria uma possibilidade real no cotidiano das empresas geradoras de resíduos, das que realizam análises e das empresas que tratam os efluentes.

Em tempos de mudanças climáticas profundas, grandes períodos de escassez e quedas recordes dos níveis das reservas de abastecimento hídrico, a importância do tratamento dos efluentes ganham cada vez mais destaque e os métodos de tratamento são constantemente questionados quanto a sua eficácia e viabilidade. Os dejetos da indústria farmacêutica de pequeno e médio porte ainda carecem de mais estudos que permitam melhor identificação dos compostos descartados e dos impactos destes compostos sobre o ecossistema e ainda poderão nortear, de forma mais eficaz, a escolha dos métodos de tratamento.

A importância dos testes rápidos fica fortalecida e a praticidade do NGTOX foi determinante na obtenção dos dados. Porém, com a análise dos resultados, a diferença comportamental apresentada entre os setores da farmácia de manipulação sugere que, de acordo com a substância a ser analisada, somente a análise via NGTOX não nos permite emitir risco de alerta ambiental, fazendo-se necessário uma avaliação complementar como, por exemplo, o ensaio de EGF ou Extração de Clorofila. A exposição crônica e a utilização de outros métodos consagrados na identificação de ecotoxicidade, como a utilização de outros biomarcadores como os microcrustáceos Daphnias ou de peixes, seriam análises de grande importância pois poderiam confrontar os dados obtidos e contribuir para o estudo do risco ambiental dos efluentes brutos dos produtores de medicamentos de pequeno e médio porte. 


\section{REFERÊNCIAS}

BALEST, L.; LOPEZ, A.; MASCOLO, G.; DI LACONI, C. Removal of endocrine disrupter compounds from municipal wastewater using na aerobic granular biomass reactor. Biochemical Engineering Journal, v. 41, p. 288-294, 2008. http://dx.doi.org/ 10.1016/j.bej.2008.05.015

BELSHE, E. F.; DURAKO, M. J.; BLUM, J. E. Photosynthetic Rapid Light Curves (RLC) oh Thalassia Testudinum exhibit Diurnal Variation. Journal of Experimental Marine Biology and Ecology, v. 342, p. 253-268, 2007. http://dx.doi.org/10.1016/ j.jembe.2006.10.056

BRASIL. Agência Nacional de Vigilância Sanitária. Anvisa e ABDI discutem descarte de resíduos de medicamentos. 27 nov. 2012. Disponível em http://portal.anvisa. gov.br/wps/content/anvisa+portal/anvisa/sala+de+imprensa/menu+noticias+anos/2012+ noticias/anvisa $+\mathrm{e}+\mathrm{abdi}+$ discutem + descarte $+\mathrm{de}+$ residuos + de + medicamentos. Acesso em: 10 jul. 2014.

BRASIL. Lei $N^{\circ}$ 9.433, de 8 de Janeiro de 1997. Institui a Política Nacional de Recursos Hídricos, cria o Sistema Nacional de Gerenciamento de Recursos Hídricos, regulamenta o inciso XIX do art. 21 da Constituição Federal, e altera o art. $1^{\circ}$ da Lei $\mathrm{n}^{\circ} 8.001$, de 13 de março de 1990, que modificou a Lei ${ }^{\circ} 7.990$, de 28 de dezembro de 1989. Diário Oficial [da] União, Brasília, DF, 09 jan. 1997. Disponível em http://www.planalto.gov.br/ccivil_03/leis/19433.htm. Acesso em: 10 jul. 2014.

BRUNTON, L. L.; CHABNER, B. A.; KNOLLMANN, B. C. Goodman \& Gilman: as bases farmacológicas da terapêutica. 12. ed. Rio de Janeiro: McGraw-Hill, 2012. 2.112 p.

CHECCUCI, A.; COLOMBETTI, G.; FERRARA, R.; LENCI, F. Action spectra for photoaccumulation of green and colorless Euglena: evidence for identification os receptor pigments. Photochemistry and Photobiology, v. 23, p. 51-54, 1976. http://dx.doi.org/10.1111/j.1751-1097.1976.tb06770.x

CORDEIRO, D. Uso de bioindicador de efeito endócrino e validação do método para determinação de hormônios na água da represa municipal de São José. 2009. Dissertação (Mestrado em Química Analítica) - Instituto de Química de São Carlos, Universidade de São Paulo, São Carlos, 2009.

CRUZ, L. H. da; HENNING, F. G.; SANTOS, A. B. dos; PERALTA-ZAMORA, P. Degradação fotocatalítica de Sulfametoxazol, Trimetropina e Diclofenaco em solução aquosa. Quimica Nova, v. 33, n. 6, p. 1270-1274, 2010. http://dx.doi.org/ $10.1590 /$ S0100-40422010000600010

D’ASCENZO, G.; DI CORCIA, A.; GENTILI, A.; MANCINI, R.; MASTROPASQUIA, R.; NAZZARI, M. et al. Fate of Natural Estrogen Conjugates in Municipal Sewage Transport and Treatment Facilitis. The Science of the Total Environment, v. 302, p. 199-209, 2003. http://dx.doi.org/10.1016/S0048-9697(02)00342-X

EKELUND, N. G. A.; NILSSON, L. Effects of estrogenic substances on the movement of Euglena Gracilis Verh. In: INTERNATIONAL ASSOCIATION OF THEORETICAL AND APPLIED LIMNOLOGY, 30., 2007, Montreal. Proceedings... Stuttgart, 2008. p. 357-359. 
ERZINGER, G. S.; HÄDER, D. P.; DEL CIAMPO, L. F. Equipamento e processo para análise de toxicidade em sistemas aquáticos. BR PI 0000221105523696. 2011.

ESPLUGAS, S.; BILA, D. M.; KRAUSE, L. G. T.; DEZOTTI, M. Ozonation and advanced oxidation technologies to remove endocrine disrupting chemicals (EDCs) and pharmaceuticals and personal care products (PPCPs) in water effluents. Journal of Hazardous Materials, v. 149, p. 631-642, 2007. http://dx.doi.org/10.1016/ j.jhazmat.2007.07.073

GONZÁLEZ-PLEITER, M.; GONZALO, S.; RODEA-PALOMARES, I.; LEGANÉS, F.; ROSAL, R.; BOLTES, K. et al. Toxicity of five antibiotics and their mixtures towards photosynthetic aquatic organisms: Implications for environmental risk assessment. Water Research, v. 47, n. 6, p. 2050-2064, 2013. http://dx.doi.org/10.1016/j. watres.2013.01.020

HÄDER, D-P.; LEBERT, M. Real time computer controlled tracking of motile microorganisms. Photochemistry and Photobiology, v. 42, p. 509-514, 1985. http://dx.do.org/10.1111/j.1751-1097.1985.tb01602.X

HUGGETT, D. B.; BROOKS, B. W.; PETERSON, B.; FORAN, C. M.; SCHLENK, D. Toxicity of select beta adrenergic receptor-blocking pharmaceuticals ( $\beta$-blockers) on aquatic organisms. Archives of Environmental Contamination and Toxicology, v. 43, n. 2, p. 229-35. 2002. http://dx.doi.org/10.1007/s00244-002-1182-7

KOCK-SCHULMEYER, M.; GINEBREDA, A.; POSTIGO, C; LOPEZ-SERNA, R.; PEREZ, S.; BRIX, R. et al. Wastewater reuse in Mediterranean semi-arid areas: The impact of discharges of tertiary treated sewage on the load of polar micro pollutants in the Llobregat river (NE Spain). Chemosphere, v. 82, p. 670-678, 2011. http://dx.doi.org/10.1016/j.chemosphere.2010.11.005

KOLPIN, D. W.; SKOPEC, M.; MEYER, M. T.; FURLONG, E. T.; ZAUGG, S. D. Urban contribution of pharmaceuticals and other organic wastewater contaminants to streams during differing flow conditions. Science of The Total Environment, v. 328, n. 1-3, p. 119-130, 2004. http://dx.doi.org/10.1016/j.scitotenv.2004.01.015

MIAO, X.; KOENIG, B.G.; METCALFE, C. D. Analysis of acidic drugs in the effluents of sewage treatment plants using liquid chromatography-electrospray ionization tandem mass spectrometry. Journal of Chromatography A, v. 952, p. 139-147, 2002. http://dx.doi.org/10.1016/S0021-9673(02)00088-2

PINTO, L. H. Estudo das alterações promovidas pelos Hormônios $17 \boldsymbol{\alpha}$ etinilestradiol e $17 \beta$ etinilestradiol na atividade fotossintética e no comportamento das algas do gênero Euglena gracilis. 2002. Dissertação (Mestrado em Saúde e Meio Ambiente) Universidade da Região de Joinville, Joinville, 2012.

PROIA, L.; OSORIO, V.; SOLEY, S.; KÖCK-SCHULMEYER, M.; PÉREZ, S.; BARCELÓ, D. et al. Effects of pesticides and pharmaceuticals on biofilms in a highly impacte driver. Environmental Pollution, v. 178, p. 220-228, 2013. http://dx.doi.org/10.1016/j. envpol.2013.02.022

RAIMUNDO, C. C. M. Contaminantes emergentes em água tratada e seus mananciais: sazonalidade, remoção e atividade estrogênica. 2011. 172f. Tese (Doutorado em Química) - Instituto de Química da UNICAMP, São Paulo, 2011. 
REVIERS, B. de. Biologia e filogenia das algas. Porto Alegre: Artmed, 2006. 280p.

RUDDER, J.; WIELE, T. V.; DHOOGE, W.; COMHAIRE, F.; VERSTRAETE, W. Advanced water treatment with manganese oxide for the removal of $17 \alpha$ ethynylestradiol (EE2). Water Research, v. 38, p. 184-192, 2004.

SANTOS, L.; ARAÚJO, A. N.; FACHINI, A.; PENA, A.; DELERUE-MATOS, C., MONTENEGRO, M. C. B. S. M. Ecotoxicological aspects related to the presence of pharmaceuticals in the aquatic environment. Journal of Hazardous Materials, v. 175, p. 45-95, 2010. http://dx.doi.org/10.1016/j.jhazmat.2009.10.100

UEDA, J.; TAVERNARO, R.; MAROSTEGA, V.; PAVAN, W. Impacto ambiental do descarte de fármacos e estudo da conscientização da população a respeito do problema. Revista Ciências do Ambiente, v. 5, n. 1, 2009.

VALCÁRCEL, Y.; GONZÁLEZ ALONSO, S.; RODRIGUEZ-GIL, J. L.; ROMO, R.; GIL, A.; CATALÁ, M. Analysis of the presence of cardiovascular and analgesic/antiinflammatory/antipyretic drugs in fluvial and drinking water of the Madrid Region in Spain. Chemosphere, v. 82, n. 7, p. 1062-1071, 2011a. http://dx.doi.org/10.1016/j. chemosphere.2010.10.041

VALCÁRCEL, Y.; GONZÁLEZ ALONSO, S.; RODRIGUEZ-GIL, J.L.; ROMO R; GIL, A; CATALÁ, M. Detection of pharmaceutically active compounds in the rivers and tap waterof the Madrid Region (Spain) and potential ecotoxicological risk. Chemosphere, v. 84, p. 1336-1348, 2011b. http://dx.doi.org/10.1016/j.chemosphere.2011.05.014

VAN DER VEN, K.; KEIL, D.; MOENS, L.; HUMMELEN, P. V.; VAN REMORTEL, P. et al. Effects of antidepressant mianserin in zebrafish: molecular markers of endocrine disruption. Chemosphere, 65: 1836-45. 2006. http://dx.doi.org/10.1016/j.chemosphere. 2006.03.079

ZUCCATO, E.; CASTIGLIONI, S.; FANELLI, R.; REITANO, G.; BAGNATI, R.; CHIABRANDO, $C$. et al . Pharmaceuticals in the environemnt in Italy: causes, occurrence, effects and control. Environmental Science and Pollution Research, V. 13, n. 1, p. 15-21, 2006. http://dx.doi.org/10.1065/espr2006.01.004 